\title{
La modélisation graphique de phénomènes émergents pour répondre aux besoins de la prospective
}

Graphic modeling of emerging phenomenon: a prospective need Modelización gráfica de fenómenos emergentes: una necesidad para la prospectiva

\section{Laure Casanova Enault et Cathy Chatel}

\section{CpenEdition}

\section{Journals}

Édition électronique

URL : http://journals.openedition.org/mappemonde/2264

DOI : $10.4000 /$ mappemonde.2264

ISSN : 1769-7298

\section{Éditeur}

UMR ESPACE

\section{Référence électronique}

Laure Casanova Enault et Cathy Chatel, «La modélisation graphique de phénomènes émergents pour répondre aux besoins de la prospective », Mappemonde [En ligne], 119 | 2017, mis en ligne le 01 janvier 2017, consulté le 02 juillet 2020. URL : http://journals.openedition.org/mappemonde/2264 ; DOI : https://doi.org/10.4000/mappemonde.2264

Ce document a été généré automatiquement le 2 juillet 2020 


\title{
La modélisation graphique de phénomènes émergents pour répondre aux besoins de la prospective
}

\author{
Graphic modeling of emerging phenomenon: a prospective need \\ Modelización gráfica de fenómenos emergentes: una necesidad para la \\ prospectiva
}

Laure Casanova Enault et Cathy Chatel

\section{NOTE DE L'AUTEUR}

Les scénarios de prospective ayant été réalisés en 2012 et la modélisation graphique en 2013, ils se fondent sur le découpage régional antérieur à la réforme territoriale de 2015.

1 Si les chorèmes de R. Brunet sont une grammaire utile pour comprendre l'organisation, le fonctionnement voire, l'évolution des espaces, leur usage se révèle parfois ardu lors de la confrontation des modèles à la complexité du réel. Compte tenu du nombre réduit de chorèmes et de leur charge théorique, il convient d'interpréter puis de sélectionner les traits saillants des phénomènes pour en dégager les structures et dynamiques associées, ce qui peut nécessiter d'importantes analyses en amont de la modélisation. De plus, certains phénomènes - en particulier lorsqu'ils sont nouveaux - renvoient à des processus qui ne sont pas toujours intégrés dans la table initiale des chorèmes.

2 La modélisation graphique des scénarios prospectifs Territoire Durable 2030 (issus d'un programme du MEDDE) interroge les possibilités ofFertes par la méthode de modéliser deux thématiques jusque-là peu appréhendées par les chorèmes et qui ont émergé avec la réflexion sur le développement durable : l'environnement et la gouvernance. 
3 Selon quelles modalités ces thématiques peuvent-elles être prises en compte en modélisation graphique? Inversement, comment la méthode de modélisation graphique utilisée dans une finalité un peu différente de celle pour laquelle elle a été élaborée initialement, à savoir modéliser l'évolution de territoires selon des scénarios de prospective, permet d'interpréter sous un regard nouveau l'environnement et la gouvernance?

\section{Temps, échelles, dimension fonctionnelle des espaces : les enrichissements de la table des chorèmes depuis 1986}

4 Le projet scientifique associé à la construction de la table des chorèmes inclut la possibilité de l'enrichir (Brunet, 1986). Temps, échelles, dimension fonctionnelle des espaces méritent en efret d'être mieux pris en compte par la méthode. Bien que le temps soit présent dans les modèles graphiques à travers un jeu de chorèmes figurant des processus, la déclinaison des temporalités multiples (Durand-Dastès, 1999; Elissalde, 2000) qui animent l'espace géographique fait défaut ${ }^{1}$. Si les chorèmes peuvent également être utilisés pour rendre compte d'organisations spatiales à différentes échelles géographiques, il est en revanche difficile de les employer pour traduire des processus multi-niveaux. Enfin, dans l'objectif de mieux comprendre les enjeux spécifiques de territoires locaux ou régionaux, certains auteurs ont enrichi la grille de chorèmes de la dimension fonctionnelle des territoires pour ensuite développer une modélisation graphique de leur espace d'étude.

$5 \quad$ Les travaux réalisés pour mieux intégrer temps, échelles et dimension fonctionnelle des territoires ces dernières années ouvrent la voie pour un possible élargissement du spectre des phénomènes interprétés par modélisation graphique comme cela est attendu en prospective.

6 Nous portons ici une attention particulière à la dimension fonctionnelle des territoires entendue au sens des actions et des usages de l'espace, dimension qui met en évidence les «relations entre les transformations socio-économiques et les dynamiques de territoires" (Bonin, 2001). En efFet, certaines pressions sur la ressource environnementale ainsi que la gouvernance et l'administration des territoires peuvent relever de cette dimension peu explorée en chorématique. Des auteurs ont déjà souligné les difficultés de représentation de l'impact de nouveaux usages de l'espace : "les processus liés à la maîtrise de l'espace " figurant dans la table des chorèmes (" appropriation, gestion, partition, drainage, irrigation, conquête ») ne suffisent pas (Brunet, 1986). Parmi les thèmes analysés au prisme de la chorématique, on trouve ceux relatifs au monde rural à travers les activités, les usages et les conflits qui lui sont spécifiques (Bonin, 2001; Michel et al., 2001; Cheylan et al. 1990), ceux relatifs au développement et à la gestion territoriale (Lardon, 2002) ou encore plus récemment les fonctions de la ville dans une dimension chronochorématique (Rodier et al., 2010-2014). Or, la prise en compte de la dimension fonctionnelle en modélisation graphique s'expose à deux écueils. D'une part, le risque est de corrompre la démarche de modélisation par le recours à la schématisation graphique, laquelle ne se réfère pas à une "grammaire spatiale " pour opérer des choix graphiques, ni à des règles de combinaison. La sémiologie cartographique peut en efFet apparaître utile pour 
représenter la dimension fonctionnelle des territoires. Pourtant, les règles d'usage dans ce domaine ne constituent pas un référentiel théorique équivalent à la table des chorèmes. D'autre part, le risque est de complexifier les modèles graphiques en résultat et donc de brouiller le message. En efFet, la dimension fonctionnelle est introduite à la suite de la modélisation des grands principes structurant le territoire. De plus, elle nécessite d'introduire un certain niveau de détails pour en comprendre les efFets (ce qui est peu compatible avec le principe d'abstraction de la modélisation graphique) alors même que plusieurs types d'activités et d'usages s'entremêlent sur un espace donné. Comme dans le cas des cartes de synthèse, la contrainte de lisibilité des modèles finaux liée à la superposition d'informations devient d'autant plus forte.

\section{La modélisation graphique de phénomènes émergents en question}

7 Le programme Territoire Durable $2030^{2}$ mené par le MEDDE en 2012 repose sur trois études réalisées dans une première étape : les études Aqua 2030 (Sala et al., 2013) et Biodiversité 2030 (Sala, 2013) ont été reprises dans une étude plus compréhensive, Territoire durable 2030, proposant des scénarios évaluant la durabilité des territoires en France à l'horizon 2030 (BIPE, 2012). La seconde étape a consisté en la modélisation spatiale de trois scénarios pour laquelle le MEDDE a fait appel à deux bureaux d'étude, Ecovia et Gaïago, et à l'UMR Espace (UMR Espace et al., 2013). Le scénario tendanciel, des territoires leviers, sera pris en exemple dans cet article.

Résumé des idées directrices du scénario des Territoires Leviers

Les scénarios Territoire Durable 2030 se distinguent par a) le poids de l'économie, b) l'importance accordée aux richesses naturelles, humaines et foncières, c) le système de gouvernance d) la dimension sociale et sociétale. Le scénario des « territoires leviers » constitue le scénario tendanciel. La France de 2030 est constituée de territoires qui gagnent le pari de la mondialisation, et de territoires qui perdent. Une gouvernance multi-échelle plus affirmée régule l'économie et la société : les territoires se restructurent autour des métropoles et des régions et l'ouverture internationale favorise les régions les plus connectées. L'extension des agglomérations se poursuit tandis que l'agriculture reste divisée entre une production intensive et une agriculture respectueuse des équilibres écologiques. Les pressions sur l'environnement se renforcent.

$8 \mathrm{Au}$ cours de ces deux étapes, de nombreuses variables (en particulier démographiques, socio-économiques et environnementales) ont été analysées, cartographiées et modélisées. Parmi ces différentes approches des scénarios, la modélisation graphique constitue un "référentiel spatial» utile pour associer des données hétérogènes peu compatibles (Brau, 2002) et communiquer visuellement les trajectoires des territoires.

9 La modélisation graphique permet en efret de dégager les structures et dynamiques fondamentales d'un espace pour en comprendre l'organisation et le fonctionnement. Toutefois, dès lors que l'objectif de la modélisation devient celui de comprendre les possibilités d'évolution des territoires, des dimensions qui ne sont pas essentielles à la compréhension de son organisation et de son fonctionnement dans le présent mais qui pourraient le devenir dans le futur sont à intégrer. La modélisation prospective 
implique de considérer comment des phénomènes secondaires, périphériques, émergents à un temps donné pourraient se révéler déterminants dans le futur. C'est l'hypothèse faite dans les scénarios Territoire Durable 2030 à travers la prise en compte de la ressource environnementale ainsi que la maîtrise des territoires par une gouvernance donnée. Elles constituent deux variables clés de la prospective Territoire Durable 2030. Comment les intégrer dans les modèles graphiques de 2010 et dans les scénarios pour 2030 ?

La modélisation graphique de ces scénarios a ainsi représenté trois enjeux.

Le premier enjeu souligne la singularité de la modélisation graphique de la dimension fonctionnelle des territoires en prospective. Suivant la démarche commune, la caractérisation de la dimension fonctionnelle des territoires intervient lors de la composition du modèle final, c'est-à-dire au moment de la prise en compte des contingences locales. En prospective, cette phase doit être introduite en amont car la dimension fonctionnelle, d'autant plus lorsqu'il s'agit de nouvelles activités, constitue un facteur explicatif de l'organisation des territoires et de leur évolution ${ }^{3}$ capable d'orienter de nouvelles trajectoires.

Le second enjeu est de modéliser les transitions menant de 2010 à 2030, c'est-à-dire les phénomènes géographiques majeurs qui interviennent dans les processus de changement des structures et dynamiques des thèmes modélisés en 2010. En efFet, à partir des scénarios narratifs, les idées forces de chaque scénario ont été sélectionnées et traduites spatialement selon quatre thèmes qui varient entre 2010 et 2030 selon différents scénarios: les réseaux urbains, les axes et les flux, la gouvernance et l'environnement. Pour modéliser les transitions entre 2010 et 2030, trois étapes de modélisation graphique se sont succédé: la sélection des indicateurs clefs, leur spatialisation, l'extraction des objets spatiaux structurants. Une autre étape de modélisation, liée à l'application de la démarche à un exercice de prospective, a consisté à reconnaître parmi ces objets ceux capables d'enclencher les dynamiques en jeu dans les scénarios de 2030.

Enfin, le troisième enjeu a été de découvrir des structures et dynamiques spatiales qui relèvent des processus d'adaptation aux défis du développement durable. En ce qui concerne la dimension environnementale, ces structures et dynamiques sont interprétées au travers des potentiels et qualités conférés aux territoires d'un côté, et des pressions, de la vulnérabilité qui les afFectent d'un autre côté. Des chorèmes originaux sont ainsi produits pour traduire les processus de fragmentation, dégradation, conflit, ponction, repli ou encore de ponction de la ressource environnementale (figure 2). En ce qui concerne la gouvernance des territoires, les relations multi-niveaux sont intégrées à la modélisation graphique pour caractériser la dimension partagée du pouvoir appelée dans les principes du développement durable.

\section{De nouveaux chorèmes pour rendre compte des mécanismes de pression sur la ressource environnementale}

11 La prégnance des enjeux environnementaux actuels et annoncés doublée de la décision gouvernementale d'opérer une nouvelle transition écologique et énergétique ${ }^{4}$ explique 
la réflexion approfondie qu'a suscitée la question environnementale dans les scénarios Territoire Durable 2030.

La difficulté est de rendre compte des mécanismes géographiques liés à la dimension environnementale qui fondent les trajectoires à 2030 et sont déjà observables dans la configuration spatiale modélisée en 2010. Elle a été résolue en modélisant la ressource environnementale en termes de potentiels et de pressions. Il s'agit aussi d'éviter l'écueil propre à toute modélisation d'un espace à l'échelle macro géographique, à savoir la trop grande simplification des phénomènes qui peut altérer la qualité du modèle. En efFet, la modélisation implique un niveau de généralisation qui est d'autant plus élevé que l'échelle géographique considérée est petite et le champ de modélisation resserré. Nombre de modélisations graphiques se réalisent à l'échelle d'États (Brunet, 1986 ; Reynaud, 1992 ; Théry, 1986) ou de continents (Grataloup, 1996) mais se fondent sur des structures premières dans l'organisation des espaces, comme les structures urbaines, les discontinuités dues aux reliefs ou au climat. En revanche, introduire la décomposition des structures élémentaires d'une dimension "moins fondamentale " dans l'organisation du territoire à l'échelle de la France augmente le risque de déséquilibre entre généralisation et prise en compte des spécificités du phénomène considéré. C'est pourquoi, une étape transitoire de schématisation graphique est insérée afin de mieux repérer au travers des données environnementales localisées, les grandes structures et discontinuités.

La modélisation graphique des enjeux liés à l'environnement, du point de vue de la biodiversité et de la ressource en eau en particulier, s'est réalisée en deux étapes : la modélisation graphique de la ressource et des pressions environnementales en 2010, puis en 2030.

Pour mettre en évidence la ressource environnementale potentielle à l'échelle nationale (figure 1), un détour par la géographie physique a été nécessaire. À partir de l'analyse de cartes thématiques réalisées en particulier par l'IFN (Inventaire Forestier National) et le BRGM (Bureau de Recherches Géologiques et Minières), l'explication de la répartition géographique de la ressource environnementale potentielle fait appel à deux chorèmes de la table élémentaire de Brunet: la structure en gradient et l'interface/rupture. D'une part, elle permet de révéler les lignes de force de la géographie physique du territoire français: les milieux ouverts, dont la répartition correspond principalement aux espaces de faible altitude, sont les moins favorables à la biodiversité, tandis que les milieux semi-ouverts valorisés en vignobles ou en bocages, et les milieux fermés, correspondant aux forêts, constituent les principaux territoires qui favorisent la biodiversité. D'autre part, les grandes structures de la géographie physique du territoire permettent de révéler la distribution des précipitations efFIcaces sur la façade océanique et sur les reliefs qui traduisent les espaces d'intérêt pour la réserve en eau de surface et en eau souterraine. 
Figure 1. Modélisation graphique de la ressource environnementale potentielle dans la France de 2010

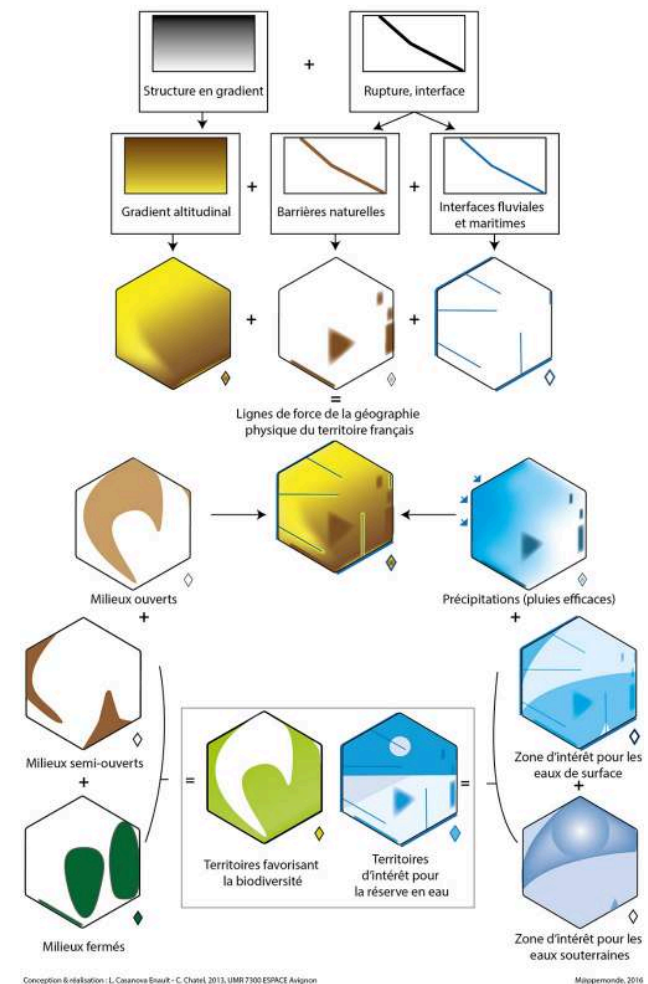

Conception et réalisation : L. Casanova Enault - C. Chatel, 2013, UMR 7300 ESPACE Avignon

L'explication de l'évolution de l'état de la ressource environnementale nécessite le recours à des chorèmes originaux. Ainsi, quatre processus rendent compte de la pression environnementale qui influence l'évolution du territoire de 2010 à 2030 : la fragmentation, la ponction ou le prélèvement, les dynamiques contradictoires, notamment pour l'appropriation de la ressource, et enfin la dégradation ou l'érosion de la ressource.

Ces chorèmes mettent en évidence les pressions liées aux conflits d'usage et d'appropriation du sol (figure 2) et qui sont en partie déduites de la confrontation entre la ressource potentielle, localisée, et les autres éléments structurants du territoire (en particulier de nature urbaine). Différentes dynamiques d'altération de la ressource (consommation en eau, activité industrielle, agriculture intensive...) aFFectent la biodiversité et les réserves en eau selon des modalités et des intensités variables en fonction des scénarios : on aboutit par exemple, à des cas de fragmentation des habitats sous l'erfet de la présence des principaux axes de communication, à des conflits d'usage du sol exacerbés dans l'espace périurbain ou à la dégradation de la qualité des sols liée à l'agriculture intensive (exemple illustré sur la figure 3). 
Figure 2. Les pressions sur la ressource environnementale

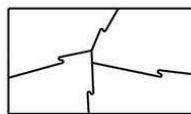

Fragmentation

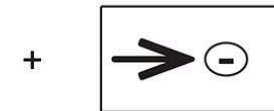

Ponctionnement

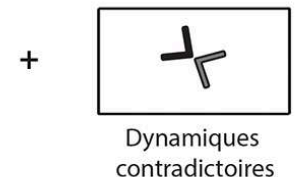

contradictoires

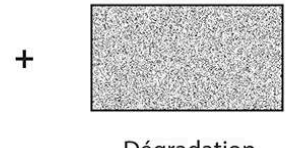

Dégradation

Figure 3. Exemple d'étape intermédiaire de modélisation des pressions sur la ressource environnementale en 2010

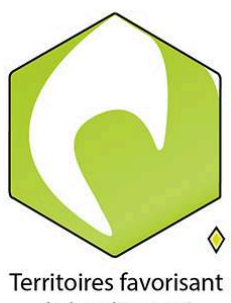
la biodiversité

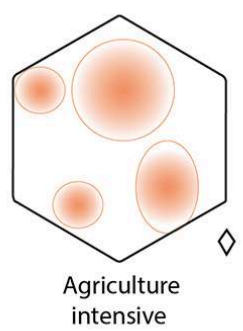

intensive

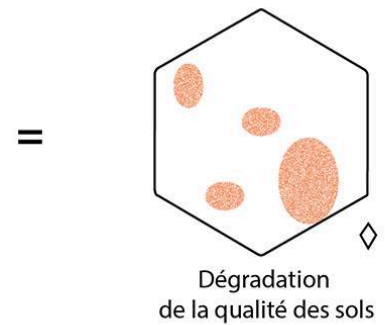

M@ppemonde, 2016

Conception et réalisation : L. Casanova Enault - C. Chatel, 2013, UMR 7300 ESPACE Avignon

Dans le scénario des territoires leviers (figure 4), la biodiversité progresse avec le développement de l'agriculture biologique, et diminue sous l'efFet des impacts du changement climatique « dans les zones les plus exposées au climat comme les espaces littoraux - mobilité du trait de côte -, la haute-montagne et les grands massifs forestiers" (BIPE, 2012). Pourtant, les pressions reconnues en 2010 vont en partie s'intensifier. C'est le cas de la fragmentation des habitats qui s'amplifie à la fois sous l'erfet de la démultiplication des infrastructures de transport, de la décentralisation des politiques publiques environnementales et de la poursuite de la périurbanisation, trois facteurs identifiés dans la modélisation graphique d'ensemble des scénarios. La périurbanisation a aussi pour conséquence d'aggraver les conflits d'usage du sol dans les périphéries urbaines. Le développement des bonnes pratiques dans le domaine de l'environnement décrit dans ce scénario ne suffit donc pas à limiter les pressions sur la biodiversité en 2030. Leur intensification s'explique par la « perception des ressources naturelles comme des ressources économiques" ainsi que par "l'anthropisation des milieux » (BIPE, 2012). 
Figure 4. Les pressions sur la biodiversité en 2010 et dans le scénario 2030 des territoires leviers

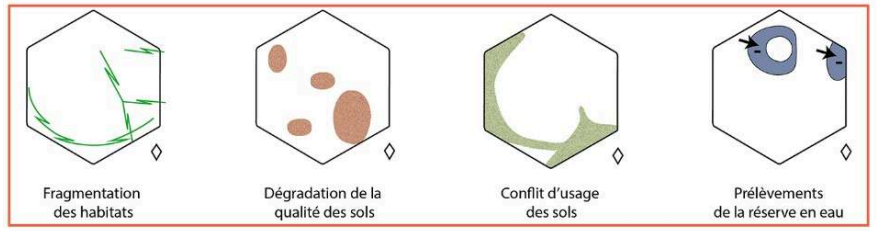

Pressions en 2010

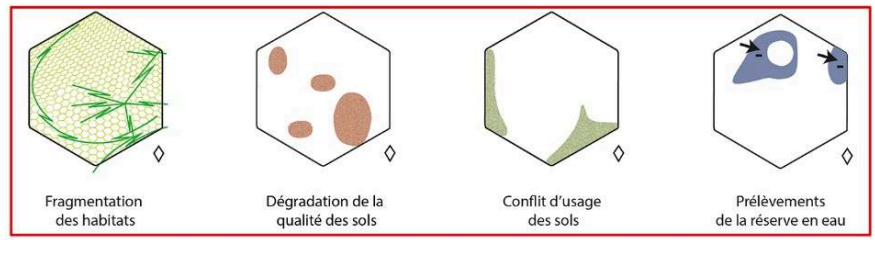

$=$

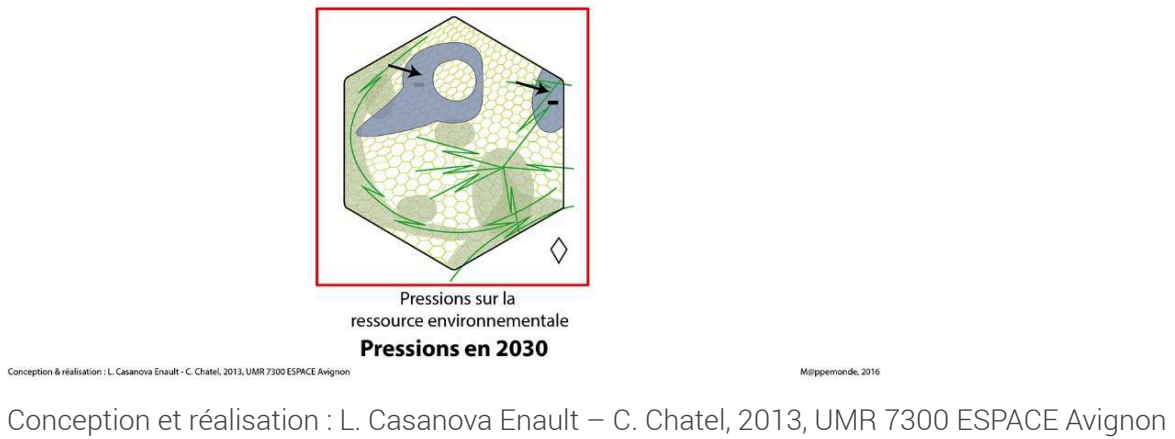

18 Ainsi, la décomposition des processus élémentaires qui interviennent dans la dynamique de la ressource environnementale permet d'expliciter les facteurs environnementaux de changement des territoires dans les scénarios. La méthodologie est conforme à celle de toute modélisation graphique. Toutefois, par rapport à la table des chorèmes initiale, les processus en jeu sont à la fois précisés (possibilité ici de signaler non seulement une diminution de la ressource mais comment elle s'opère) et adaptés (en mobilisant le concept d'érosion par exemple) à la thématique environnementale.

\section{Rendre compte de la gouvernance et de l'administration territoriales par modélisation graphique : centres, mailles et relations multi-niveaux}

19 Les modes de gouvernance et le rôle des institutions gouvernementales et administratives dans les changements territoriaux projetés en 2030 constituent un élément clé des scénarios prospectifs Territoire durable 2030.

La notion de gouvernance est comprise ici comme le versant social du développement durable (ONU, 2012), acception en rupture avec la philosophie néo-libérale associée au terme à l'origine (corporate governance). Ce référentiel colore ainsi positivement le terme de gouvernance, dont la signification tend à se substituer à celle de mode de gouverner (McCarney et al., 1995). En tant qu' " approfondissement de la démocratie » (Dufour, 2009), la gouvernance se fonde désormais sur les principes d'organisation décentralisée, de démocratie participative ou encore de concertation. qui sont les 
corollaires d'une gestion partagée du pouvoir entre des acteurs multiples lesquels sont associés à différents niveaux géographiques d'intervention en interaction.

Dans les travaux de prospective, le mode de gouvernance est, soit une variable de contexte (Datar, 2009), soit une variable d'ajustement dans les scénarios et il modifie alors les trajectoires identifiées (Espon Project 3.2, 2007), soit un facteur majeur de changement (BIPE, 2012). L'importance variable accordée à la gouvernance peut s'expliquer par la difficulté à identifier des indicateurs de la gouvernance et à la mesurer. Bien souvent, on mesure ${ }^{5}$ la gouvernance par son impact, sur la société et le territoire.

22 À la différence de la thématique de l'environnement, aucune expertise du programme n'a produit des indicateurs ou des cartes. La modélisation graphique a constitué une solution méthodologique pour à la fois représenter la gouvernance et formaliser de manière qualitative son impact sur les territoires. L'expression territoriale de la gouvernance correspond aux niveaux de l'action et de l'administration territoriale. Aussi, la représentation de la dimension scalaire a été particulièrement explorée.

Toutefois, les modes de gouvernance sont par nature des phénomènes a-spatiaux qui restent difficiles à représenter graphiquement, malgré les efFets territoriaux directs, très visibles qui en dépendent (poids et influence - démographiques, économiques, sociales, culturelles... - des capitales et chefs-lieux, efFet-barrière ou au contraire attractivité des frontières administratives de divers niveaux, formes et déploiement des structures urbaines suivant les modèles de développement territorial). Nous nous sommes concentrées principalement sur les structures administratives mais aussi sur les différents acteurs de la gouvernance intervenants dans les scénarios (acteurs individuels publics et privés, collectivités, intercommunalités) et sur leurs niveaux d'action. L'importance accordée aux structures administratives et à la hiérarchie des niveaux dans la modélisation s'explique par le rôle encore très prégnant et hérité de l'armature territoriale d'État en France ${ }^{6}$ qui intègre encore peu la société civile dans la conception des politiques publiques. Malgré les formes inédites de gouvernance décrites dans les scénarios ${ }^{7}$, la méthode de modélisation graphique ne peut oblitérer l'influence réelle qu'exerceront en 2030 des structures étatiques qui perdurent depuis des siècles. Il s'agit de combiner les structures spécifiques au contexte français avec les formes de gouvernance développées dans les scénarios et parfois issues de conceptions idéalisées.

24 Une des difficultés a été de représenter les différents acteurs de la gouvernance. Généralement, un niveau d'action territoriale ou l'interaction entre plusieurs niveaux bien définis est associé à un mode de gouvernance caractéristique d'un scénario. Par exemple, le scénario des «Territoires leviers » décrit une gouvernance multi-échelles fondée sur les régions, les métropoles et l'État avec une intégration croissante à l'Europe.

Les niveaux de gouvernance du territoire français en 2010 font l'objet d'un modèle graphique de référence (figure 5) à partir duquel les scénarios pour 2030 vont diverger. La démonstration utilise les chorèmes relatifs à la hiérarchie et au maillage ainsi que les principes de représentation de l'administration proposés par Christaller. Un pavage triangulaire, associant lignes et nœuds, permet de fonder des hexagones réguliers et égaux, couverture exhaustive du territoire français. Dans ce double maillage ${ }^{8}$ en triangles et en hexagones, s'imbriquent ainsi d'une part des hexagones de différentes tailles, relatifs aux strates administratives de niveaux distincts (représentées par un 
chorème original "multi-niveaux »), et d'autre part des lieux centraux correspondant aux nœuds hiérarchisés des mailles du réseau.

Les quatre niveaux géographiques intervenant dans les scénarios et auxquels sont associés un mode de gouvernance sont représentés : les métropoles, les territoires de la décentralisation, l'État, les territoires supranationaux. Ces derniers sont figurés par deux chevrons qui encadrent l'hexagone français: l'Europe et le monde. Le rôle de chacun de ces niveaux d'administration du territoire dans les modes de gouvernance et les processus territoriaux engagés est souligné graphiquement par l'épaisseur des mailles.

Trois situations théoriques sont différenciées pour mettre en évidence que le poids de chacun des niveaux diffère selon les modes de gouvernance et que, de la répartition des compétences dépendent un mode de gouvernance et un fonctionnement territorial spécifiques. Dans une intégration supranationale prépondérante, les mailles sont poreuses, les métropoles et les réseaux fondent les territoires et les flux vers l'extérieur sont intenses (figure 5-a). Dans le cadre d'un pouvoir centralisé, la capitale prime, les relations à l'intérieur du territoire national sont privilégiées et passent exclusivement par la capitale (figure 5-b). La décentralisation suppose l'assemblage de régions animées chacune par des métropoles où les flux internes à l'État sont faibles et ceux dirigés vers l'extérieur variables selon la dynamique des territoires (figure 5-c).

Ces trois modèles intermédiaires sont confrontés ensuite aux réalités du territoire français en 2010 (figure 5) lequel présente trois principales déformations par rapport au modèle théorique: la primauté de la capitale, la localisation préférentielle des grandes villes en périphérie du territoire français, et la superficie variable des régions, dilatées au Sud-Ouest, rétractées au Nord-Est. L'administration territoriale se caractérise par un poids des régions et de l'Europe plus faible que celui de l'État. Ainsi, la modélisation de la gouvernance s'illustre par ses impacts sur le territoire déduits à partir de la confrontation aux autres structures du territoire. Par exemple, le réseau urbain est en partie le fruit de la hiérarchie des chefs-lieux définis dans l'armature administrative: les trajectoires des villes sont partiellement déterminées ou au contraire totalement indépendantes de leurs fonctions administratives. De même, la proximité des frontières françaises a pu influencer les dynamiques spécifiques identifiées parmi les grandes villes françaises éloignées de Paris. Ainsi, les modélisations graphiques réalisées pour les deux thématiques relatives aux réseaux urbains et aux axes et flux ${ }^{9}$ sont largement exploitées pour découvrir les déformations des modèles intermédiaires de la gouvernance et établir le modèle final de 2010. 
Figure 5. Modélisation graphique de l'administration et de la gouvernance des territoires en 2010

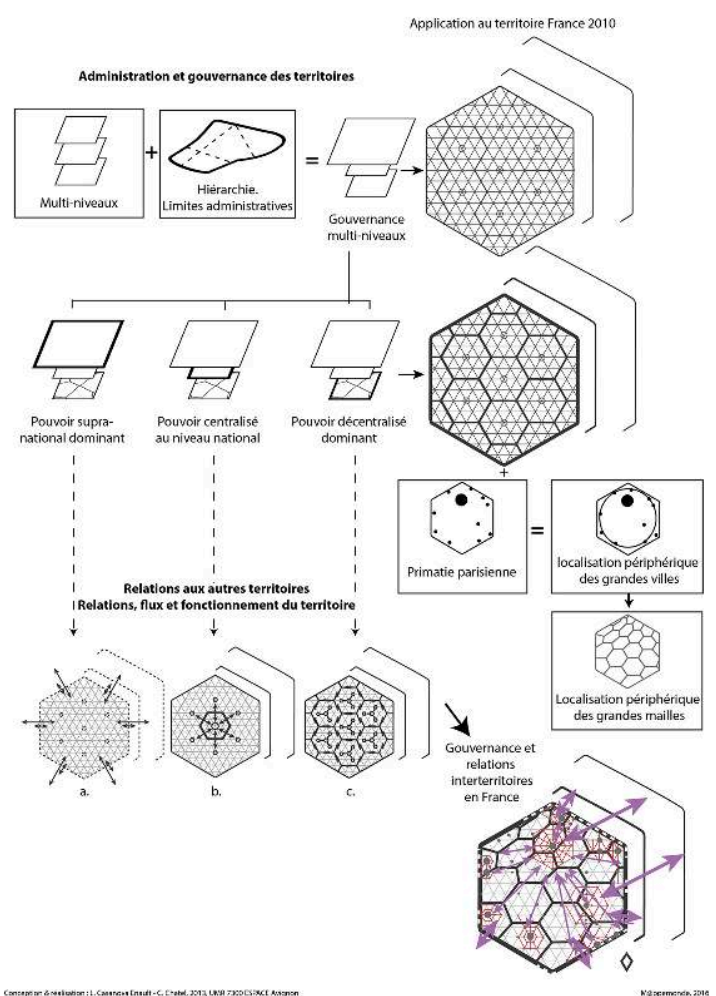

Conception et réalisation : L. Casanova Enault - C. Chatel, 2013, UMR 7300 ESPACE Avignon

En 2010, malgré un État fort, les métropoles et les régions à la périphérie du pays créent les conditions d'une croissance supérieure à la moyenne : leur situation favorise à la fois leur émancipation vis-à-vis de l'aire urbaine parisienne et leur intégration en Europe via un système de flux de plus en plus intenses.

Le scénario des Territoires Leviers se situe en continuité avec la situation actuelle. Dans un contexte "d'atonie économique », « l'État opte pour une gestion plus décentralisée donnant lieu à une gouvernance multi-échelles, où les régions, les métropoles et l'État [...] fixent le cap et le tempo de l'action publique ...] Dans ce scénario, les territoires les mieux placés dans la concurrence internationale se portent mieux. [...] L'objectif affiché est de doter la France de plusieurs centralités territoriales, capables de relayer la mégalopole parisienne dans la compétition économique mondiale. [...] La France de 2030 est composée d'une mosaïque de territoires dont certains sont innovants, dynamiques et très connectés à l'économie-monde, tandis que d'autres, en déclin, s'alignent avec retard sur les évolutions en cours ailleurs, et que d'autres encore surnagent» (BIPE, 2012). Sur la base du modèle théorique de 2010 (en grisé sur la figure 5) représentant les différentes structures administratives, le scénario valorise les métropoles, les régions, l'État et aussi l'Europe. L'intégration supranationale est un élément discriminant majeur qui dicte la différenciation entre les dynamiques des régions: son impact sur les éléments structurants du territoire est représenté. L'organisation des réseaux urbains et des flux met en évidence un processus où les régions et les métropoles les plus intégrées à la compétition européenne et mondiale vont s'émanciper en 2030. Les flux se réalisent entre les métropoles françaises de province et d'un côté, les régions transfrontalières et l'Europe, et d'un autre côté, Paris. Les métropoles françaises n'interagissent pas entre-elles, entretiennent des relations 
de compétition, et se développent au sein de leur propre réseau régional. L'intégration du territoire national s'afFaiblit. Par conséquent, les territoires sont confrontés à un processus de fragmentation (figure 6) représenté par un des chorèmes créés plus tôt : le chorème de la fragmentation déforme ainsi les trois modèles intermédiaires. L'afFaiblissement de l'intégration nationale au profit de l'intégration de certains territoires français dans le jeu européen et mondial, ainsi que la poursuite de la décentralisation prévue dans le scénario aboutit à deux types de fragmentation dont rend compte le modèle final. D'une part, le scénario présente une France duale opposant les régions dynamiques qui parviennent à éviter la crise par leur intégration sur les scènes européenne et mondiale, situées en périphérie du territoire, auxquelles s'ajoute Paris, et les régions à l'écart de ces nouvelles logiques souffrant de l'abandon d'un système de solidarité nationale et d'une situation centrale en France. D'autre part, la fragmentation entre les régions est soulignée: les régions qui gagnent, gagnent seules et sont en compétition entre elles. Pour ces régions, l'élargissement de leurs réseaux aux régions voisines constitue un risque d'afFaiblissement. Les régions qui perdent sont délaissées à tous les niveaux, par l'Europe, par l'État, par des régions dynamiques auxquelles elles auraient pu s'arrimer.

Figure 6. Modélisation graphique de l'administration et de la gouvernance des territoires en 2030 selon le scénario des territoires leviers

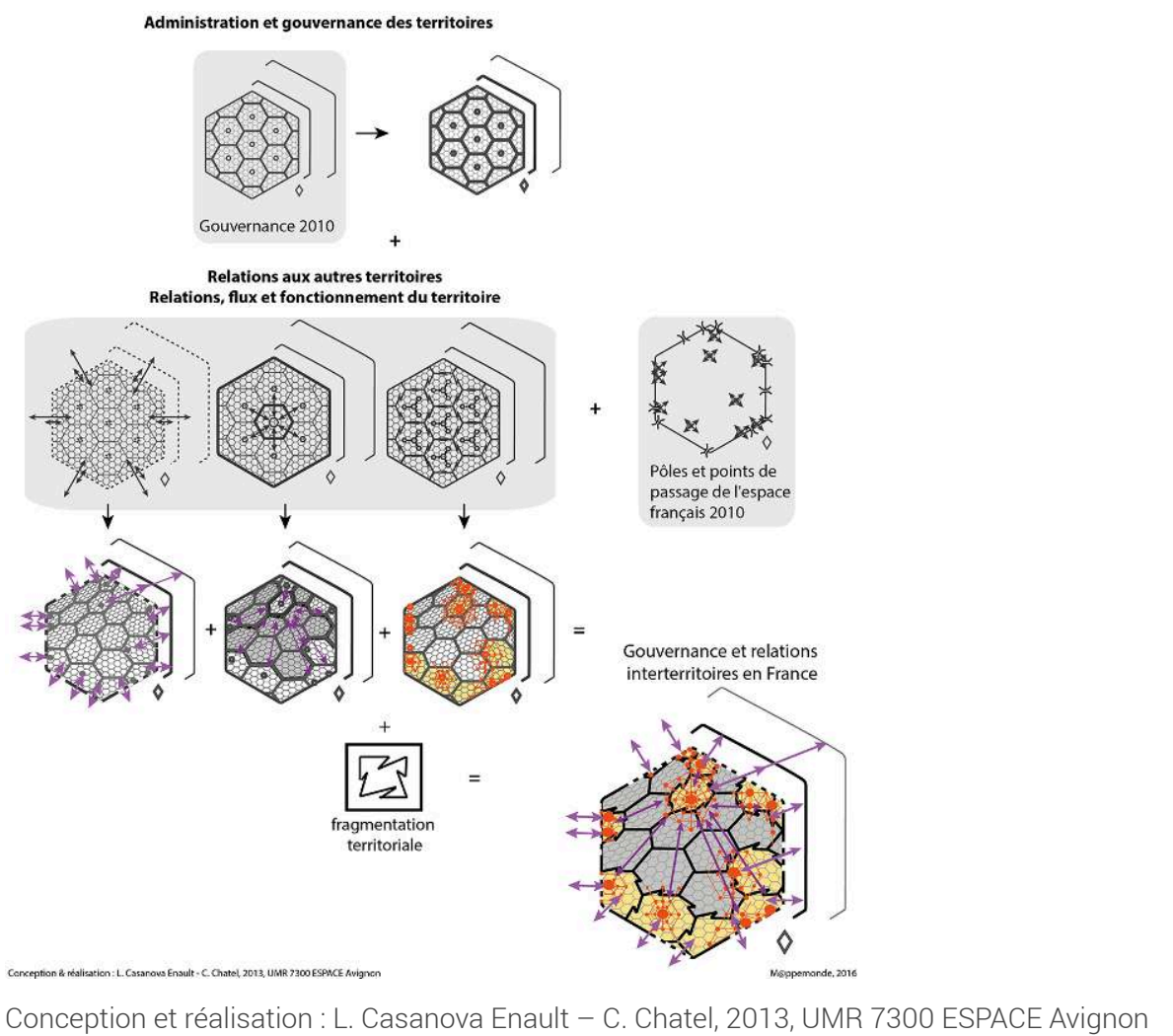

31 La prise en compte de phénomènes secondaires et/ou nouveaux, souvent relatifs à la dimension fonctionnelle des espaces, est indispensable en prospective. La modélisation graphique permet cela à condition de procéder à la décomposition des processus élémentaires qui interviennent dans la dynamique du phénomène géographique étudié. Également, afin de limiter les deux écueils cités plus haut (point 1), notre démarche a 
consisté non pas à représenter la dimension fonctionnelle des territoires, mais ce qui, à travers cette dimension, permet de comprendre leur organisation. Le premier exemple (pressions environnementales) se fonde sur des chorèmes spécifiques conçus de manière hypothético-déductive avec l'appui de matériaux collectés en amont de la modélisation (cartes en particulier). Ce référentiel nous permet de rester dans le domaine de la modélisation plutôt que celui de la schématisation. Le second exemple (gouvernance des territoires) nous a conduit à prendre de la distance avec le concept de gouvernance et toute l'amplitude de sa signification (d'ailleurs exploitée dans les scénarios narratifs) pour privilégier les conséquences spatiales qu'auraient difFérentes logiques d'administration du territoire. Ces exemples ont ainsi montré qu'il est possible d'intégrer la dimension fonctionnelle des territoires aux modèles graphiques et que cela implique d'enrichir la table initiale des chorèmes. La méthode de modélisation graphique a été exploitée ici dans sa dimension exploratoire. La prospective territoriale offre à la méthode un renouveau attendu en valorisant sa dimension heuristique, tandis que la modélisation graphique suggère de nouvelles pistes en prospective territoriale, notamment pour les géographes.

\section{BIBLIOGRAPHIE}

BIPE (2012). Territoires durables 2030 : un exercice de prospective. Rapport d'étude, MEDDE, Commissariat général au développement durable, Mission Prospective.

BONERANDI E., SANTAMARIA F. (2011). « De la gouvernance à la gouvernance territoriale : enjeu de l'européanisation de l'aménagement du territoire. Réflexions à partir d'une recherche dans le cadre de l'Observatoire en réseau de l'aménagement du territoire européen (ORATE) ». Cybergeo : European Journal of Geography, Aménagement, Urbanisme, document 522. En ligne : https:// journals.openedition.org/cybergeo/23530

BONIN M. (2001). « Nouvelles fonctions de l'agriculture et dynamiques des exploitations. Une analyse chorématiques dans les Monts d'Ardèche ». Mappemonde, $\mathrm{n}^{\circ} 62$ (2001/2), p. 11-16. En ligne : https://www.mgm.fr/PUB/Mappemonde/M201/Bonin.pdf

BRAU F. (2002). « Les chorèmes comme outil d'analyse des besoins exprimés par des gestionnaires du territoire ». Mappemonde, $n^{\circ} 68$ (2002/4), p. 7-10. En ligne : https://www.mgm.fr/PUB/ Mappemonde/M402/Brau.pdf

BRUNET R. (1986). « La carte-modèle et les chorèmes ». Mappemonde, (1986/4), p. 2-6. En ligne : https://www.mgm.fr/PUB/Mappemonde/M486/p2-6.pdf

CASANOVA L. (2010). Les dynamiques du foncier à bâtir comme marqueurs du devenir des territoires de Provence intérieure, littorale et préalpine. Éléments de prospective spatiale pour l'action territoriale. Avignon : Université d'Avignon, thèse de doctorat de géographie, $441 \mathrm{p}$.

CGDD (2013). « Territoire durable 2030. Phase 1 : Enjeux et tendances ». Études \& Documents, $\mathrm{n}^{\circ} 93$. CGDD (2012). « Territoire durable 2030. Une prospective de développement durable à l'échelle des territoires ». Le point sur, $\mathrm{n}^{\circ} 124$. 
CHEYLAN J.-P., DEFFONTAINES J.-P., LARDON S., THÉRY H. (1990). « Les chorèmes : un outil pour l'étude de l'activité agricole dans l'espace rural ? ». Mappemonde (4/1990), p. 2-4. En ligne : https:// www.mgm.fr/PUB/Mappemonde/M490/PREFACE.pdf

DATAR (2009). Territoires 2040. Revue d'étude et de prospective, Paris : DATAR, La documentation française.

DUFOUR D. (2009). « La gouvernance comme nouvelle forme de contrôle social ». Connexions, 2009/1, n 91, p. 41-54.

DURAND-DASTÈS F. (1999). « Jamais deux fois... Ou : de quelques précautions à prendre avec le temps ». Travaux de l'Institut de Géographie de Reims, n 101-104, p. 5-23.

ELISSALDE B. (2000). «Géographie, temps et changement spatial ». L'Espace Géographique, n²9, p. $224-236$

ESPON PROJECT 3.2 (2007). Scenario on the territorial future of Europe. Rapport. En ligne : https:// www.espon.eu/export/sites/default/Documents/Publications/ESPON2006Publications/ SpatialScenarios/espon3.2_60p._final_16-7-2007-c.pdf

GRATALOUP C. (1996). Lieux d'histoire. Essai de géohistoire systématique. Montpellier : Reclus ; Aubervilliers : La Documentation française, coll. « Espaces modes d'emploi », 200 p. ISBN 2-86912-065-6.

LARDON S., PIVETEAU V. (2002). « Chorèmes et diagnostics de territoire : une expérience de formation ». Mappemonde, $n^{\circ} 68$ (2002/4), p. 1-6. En ligne : https://www.mgm.fr/PUB/ Mappemonde/M402/Lardon.pdf

MCCARNEY P., HALFANI M., RODRIGUEZ A. (1995). « Towards an Understanding of Governance: The Emergence of an Idea and its Implications for Urban research in Developing Country ». In STREN R., KJELLBERG BELL J. (éd.), Urban Research in the Developing World, vol. 4, « Perspectives on the City », Toronto Center for Urban and Community Studies, University of Toronto, 420 p. ISBN 0-7727-1371-5

MICHEL C., LARDON S. (2001). «L'accès aux espaces naturels : l'apport des chorèmes à la gestion des conflits d'usage ». Mappemonde, $\mathrm{n}^{\circ} 62$ (2001/2), p. 1-5. En ligne : https://www.mgm.fr/PUB/ Mappemonde/M201/Lardon-Michel.pdf

NATIONS UNIES (ONU) (2012). L'avenir que nous voulons. Rio de Janeiro, résultats de la Conférence Rio +20 .

RODieR X., GRATAloup C., GUilloteau C. (2010-2014). « Dossier : Chrono-chorématique urbaine ». Mappemonde, $\mathrm{n}^{\circ} 100,105$ et 114. En ligne : https://mappemonde-archive.mgm.fr/ dos_chrono.html

THÉRY H. (1986). Brésil : un atlas chorématique. Brasil : um atlas coremáticor. Brazil: a chorematical atlas. Paris: Fayard/Reclus, coll. « Atlas Reclus ». 87 p. ISBN 2-213-01794-8

ZANIN C. (dir.) (2008). Les cartes de la cohésion territoriale. Paris : DIACT (Délégation interministérielle à l'aménagement et à la compétitivité), 74 p. ISBN 9782-11-098-534-7

UMR ESPACE (CNRS), GAÏAGO, ECOVIA (2013). Géoprospective : modélisation et représentation graphique des scénarios de prospective pour un territoire durable à horizon 2030. Rapport de recherche, MEDDE-Commissariat général au développement durable-Mission Prospective, $124 \mathrm{p}$.

SALA P. (2013). Biodiversité et Territoires 2030 : cinq scénarios d'évolution-synthèse de l'exercice de prospective : volets 1 et 2 . Collection « Études et documents » de la Délégation au développement 
durable (DDD) du Commissariat Général au Développement Durable (CGDD), Études \& documents, $\mathrm{n}^{\circ} 86$, juin 2013, 40 p. En ligne

SALA P., JANNES-OBER E., LAMBLIN V. (2013). Eau, milieux aquatiques et territoires durables 2030 - synthèse de l'exercice de prospective. Collection «Études et documents » de la Délégation au développement durable (DDD) du Commissariat Général au Développement Durable (CGDD), Études \& documents, $\mathrm{n}^{\circ}$ 91, août 2013, 52 p. En ligne

\section{NOTES}

1. Un article complémentaire à celui-ci et produit suite aux travaux issus du programme territoire durable 2030 est consacré à la modélisation graphique des dynamiques spatiales. Une grille chorématique des dynamiques spatiales pour expliquer l'évolution des territoires.

2. Le site internet dédié au programme présente les objectifs du projet et l'ensemble des produits qui en sont issus : rapports, cartes, datavisualisation, modélisation graphique.

3. Cet élément est discuté dans la définition du système territorial proposée par Casanova en 2010.

4. La loi relative à la transition énergétique pour la croissance verte a été adoptée le 18 août 2015. 5. Dans ce cas, plutôt que le mode de gouvernance, c'est le maillage administratif qui est évalué en termes de cohésion territoriale (Zanin, 2008) ou d'intégration d'une région frontalière (id.) à partir d'un jeu d'indicateurs principalement d'ordre économique (relatifs à la production ou aux flux). De nouvelles approches tentent de déterminer l'influence des structures administratives sur l'organisation territoriale, en termes de hiérarchie des villes, d'interaction entre les différents niveaux administratifs (Bonerandi et al., 2011). À la difficulté de mesurer la gouvernance s'ajoute celle de la représenter.

6. Les scénarios de gouvernance proposés peuvent en revanche être développés à partir d'autres formes de gouvernance, variées et bien plus flexibles, développée dans d'autres pays européens où la culture politique est bien différente.

7. Par exemple, tous les scénarios prévoient l'affaiblissement du rôle de l'État. Ainsi, le scénario des «territoires inversés » suppose l'imposition d'une gouvernance locale presque exclusive, le scénario des «territoires singuliers» se caractérise par les interventions combinées voire contradictoires d'une multiplicité d'acteurs (intercommunalités, acteurs publics et privés), tandis que le scénario des «territoires affinitaires» inscrit la gestion du territoire dans le développement de communautés diverses (réseaux sociaux, d'entreprises..).

8. Voir la définition de réseau dans Brunet R., Ferras R., Théry H. (1993). Les mots de la géographie : dictionnaire critique. Montpellier: GIP RECLUS ; Paris : la Documentation française. Les auteurs expliquent que la maille et le réseau sont les deux faces d'une même médaille.

9. Voir les rubriques 1 et 2 de la modélisation de la France en 2010. http://www.territoiredurable-2030.developpement-durable.gouv.fr/index.php/td2030/scenario/?

$\mathrm{id}=$ actuel\&page $=$ model 


\section{RÉSUMÉS}

La modélisation graphique de scénarios prospectifs développés dans le programme Territoire durable 2030 piloté par le Ministère de l'Écologie, du Développement Durable et de l'énergie (MEDDE) a été l'occasion d'interroger l'ajustement de la méthode aux besoins de la prospective. L'enjeu réside dans l'exploration des modes de représentation de deux thématiques difficile à appréhender par la méthode et qui sont pourtant devenues décisives dans la compréhension de l'évolution des territoires du fait de la généralisation du référentiel de développement durable : l'environnement et la gouvernance.

The graphic modeling of prospective scenarios developed under the Sustainable Territory 2030 program - developed by the Ministry of Ecology, Sustainable Development and Energy - ofFered an opportunity to examine the adjustment of the method to the needs of prospective representation. The challenge was to experiment new ways to represent two topics that are difFI cult to grasp with the method; but which have become decisive in the understanding of territorial evolution due to the generalization of the reference base for sustainable development: the environment and governance.

El programa de Sostenibilidad Territorial 2030, coordinado por el Ministerio de Ecología, Desarrollo Sostenible y Energía (MEDDE), ha servido de plataforma para afinar y desarrollar modelos gráficos para escenarios prospectivos. El reto consistió en explorar nuevos modos para representar el medioambiente y la gobernanza, cruciales para comprender la evolución de los territorios y el desarrollo sostenible.

\section{INDEX}

Mots-clés : environnement, gouvernance, modélisation graphique, prospective

Keywords : environment, governance, graphic modeling, prospective

Palabras claves : medioambiente, gobernanza, modelización gráfica, prospectiva

\section{AUTEURS}

\section{LAURE CASANOVA ENAULT}

Université d'Avignon, UMR ESPACE 7300 CNRS

\section{CATHY CHATEL}

UNESP Brésil 